\title{
Anticancer dendritic cell vaccines producing effective antitumor $T$ cell responses
}

\begin{abstract}
In the past two decades, immunotherapy has been investigated as a novel modality in cancer therapy. The aim of immunotherapy is augmenting body's immune system to recognize and destroy malignant cells efficiently and safely. Dendritic cell (DC)-based vaccination is one of the most important approaches of cancer immunotherapy, in which autologous ex vivo generated tumor antigen-loaded DCs are injected to cancer patients in order to augment antitumor immunity. Various DC culture conditions, tumor antigen sources, antigen loading strategies, DC maturation stimuli, and routes of vaccination have been used to increase efficacy of DC vaccines. However, therapeutic efficacy of DC vaccines is still limited in cancer patients and preparation of clinical-grade DCs should be standardized to generate immuno stimulatory DCs and to prevent development of immunosuppressive DCs. Understanding DC function in triggering adaptive immunity or induction of immunological tolerance in cancer setting may be helpful in improving therapeutic efficacy of DC vaccines. In this article, we reviewed DCs interactions with T cells, efficacy of ex vivo generated DCs in triggering/suppression of antitumor effector $\mathrm{T}$ cell responses, as well as recent findings about DC vaccinations in cancer patients.
\end{abstract}

Keywords: cancer, dendritic cell vaccines, t cells, antitumor immune responses, immuno suppression
Volume 4 Issue 3 - 2017

\author{
Samad Farashi-Bonab,' Khansari $\mathrm{N}^{2}$ \\ 'Department of Immunology, Tehran University of medical \\ Sciences, Iran \\ ${ }^{2}$ American Medical Diagnostic Laboratory, USA
}

Correspondence: Nemat Khansari, American Medical Diagnostic Laboratory, 1665 Garden Grove Blvd, Garden Grove CA 92843, USA, Tel + I (949) 228-8290, Email nkhansari928@gmail.com

Received: February 18, 2017 | Published: April 24, 2017
Abbreviations: DC, dendritic cell; APC, antigen presenting cell; PRR, pattern recognition receptor; TLR, toll like receptor; $\mathrm{MHC}$, major histocompatibility complex; $\mathrm{CD}$, cluster differentiation; LFA-3, lymphocyte-function associated antigen-3; ICAM-1, intracellular adhesion molecule-1; TCR, $\mathrm{t}$ cell receptor; CD40L, cd40 ligand; ICOS, inducible T cell costimulator; ICOS-L, inducible $\mathrm{T}$ cell costimulator ligand; IFN, interferon; GM-CSF, granulocyte macrophage-colony stimulating factor; TRANCE, tumor necrosis factor-related activation-induced cytokine; Bcl-XL, b-cell lymphomaextra large; STAT4, signal transducer and activator of transcription-4; WT1, wilms tumor antigen 1; Th, type 1 helper T cell; CTL, cytotoxic T lymphocyte; IL, interleukin; U.S. FDA, united states food and drug administration; PAP, prostatic acid phosphatase; HNSCC, head and neck squamous cell carcinoma; Treg, regulatory T cell, MART1, melanoma antigen recognized by $\mathrm{T}$ cell-1; NY-ESO-1, new york esophageal squamous cell carcinoma-1; TNF- $\alpha$, PGE2, prostaglandin E2; tumor necrosis factor-alpha; NK, natural killer; NKT, natural killer T; MIP, macrophage inflammatory protein; AICD, activation induced cell death; PD-L, programmed death-ligand; CD95L, CD95 ligand; TRAIL, TNF-related apoptosis induced ligand; CTLA-4, cytotoxic T lymphocyte antigen-4; TGF- $\beta$, transforming growth factor-beta; IDO, indoleamine 2,3-dioxygenase; NO, nitric oxide; FOXP3, forkhead box P3; Tr1, type 1 regulatory T; CCR7, chemokine receptor 7

\section{Introduction}

Traditional anticancer therapies, including surgery, chemotherapy, and radiotherapy, have long been used to treat various types of cancer. These therapeutic modalities are successful in treating various tumors and cancers which are in initial phases of development. However, efficacy of these therapies is often disappointing in advanced cancers. In the past two decades, immunotherapy has been presented as a novel therapeutic modality for cancer. The aim of immunotherapy is augmenting body's immune system to recognize and destroy malignant cells efficiently and safely. Several immune cells and their products are involved in the recognition and elimination of tumor/ cancer cells. Among them, dendritic cells (DCs) and T cells are most studied for cancer immunotherapy. Ex vivo generated DCs pulsed with tumor specific antigens can be successfully used to produce antitumor immunity as they have a central role in the induction of antigen-specific $\mathrm{T}$ cell immune responses. DCs are a heterogeneous population of cells with widespread tissue distribution and have important roles in innate immunity. DCs can also activate naïve T cells as they have a high ability to uptake antigens from the periphery and trafficking to the $\mathrm{T}$ cell areas of the peripheral lymphoid tissues such as lymph nodes, express high levels of cell surface antigen presenting molecules and several costimulatory molecules, and produce various chemokines, cytokines as well as other immunostimulatory factors. Indeed, DCs are the most potent antigen presenting cells (APCs) and function to trigger antigen-specific immune responses. ${ }^{1,2}$ This property of DCs has led to the use of DCs as cellular vaccines in order to immunize cancer patients against tumor-specific/associated antigens and to break immunological tolerance to tumor cells. ${ }^{3}$ In this article, we reviewed DC interactions with $\mathrm{T}$ cells, the major players in antigen-specific adaptive immune responses, efficiency of DC vaccines in triggering/suppression of effector $\mathrm{T}$ cell responses, and recent findings about DC vaccinations in cancer patients.

\section{Immunophenotype of DCs and their critical role in the induction of antigen-specific $T$ cell immune responses}

DCs are sentinel cells in the body. They express cell surface pattern recognition receptors (PRRs) such as TLRs, the phagocytosis receptor DEC-205, and FC $\gamma$ receptors to explore the periphery from pathogenic agents and have a high capacity to capture and process antigens. After antigen capture, DCs migrate to the peripheral lymphoid tissues and become mature. Mature DCs express high levels of cell surface antigen presenting molecules, including MHC class I, MHC class II, and CD1 molecules, co stimulatory molecules such as CD40, CD80 (B7-1), and CD86 (B7-2), and adhesion molecules such as LFA-3 (CD58) and ICAM-1 (CD54). Expression of MHC 
molecules and MHC-peptide complexes on DCs is 10-100 fold more than other professional APCs, including B cells and monocytes. ${ }^{1,46}$ These properties of DCs give them a central role in the initiation of antigen-specific $\mathrm{T}$ cell responses.

Generation of strong and durable antigen-specific $\mathrm{T}$ cell responses is necessary to achieve effective antitumor immunity. Both $\mathrm{CD} 4^{+} \mathrm{T}$ cells and $\mathrm{CD} 8^{+} \mathrm{T}$ cells are important in creation of tumor immunity. ${ }^{7-9}$ $\mathrm{T}$ cells need to receive three signals to be properly activated:

i. Antigen recognition signals elicited by binding of TCR on the cell surface of T cells to antigen presented in the context of antigen presented molecules MHC class I or class II molecules expressed on target cells or APCs,

ii. Costimulatory signals elicited by interactions of cell surface molecules CD28, CD40L, CD27, and ICOS expressed on T cells with co stimulatory molecules CD80/CD86, CD40, CD70, and ICOS-L, respectively, expressed on professional APCs, and

iii. Signals arising from attachment of soluble molecules such as cytokines to cognate receptors expressed on T cells. ${ }^{10-13}$

Thus, antigen recognition by $\mathrm{T}$ cells is not solely sufficient for $\mathrm{T}$ cell activation and signaling via co stimulatory molecules as well as signaling mediated by immuno stimulatory cytokines are important for efficient activation of $\mathrm{T}$ cells and triggering antigen specific immune responses. Indeed, antigen detection in the absent of secondary signals can lead to immunological tolerance or energy of T cells. ${ }^{1}$ DCs are crucial in this regard by providing these signals for $\mathrm{T}$ cell activation. Cytokines such as IFN- $\gamma$ and GM-CSF, secreted by T cells, activate DCs, and subsequently, DCs provide appropriate signals for activation of antigen-specific $\mathrm{T}$ cells. In addition, DCs stimulated by $\mathrm{CD} 4^{+} \mathrm{T}$ cells via CD40-CD40L interactions can more effectively present antigens to $\mathrm{CD} 8^{+} \mathrm{T}$ cells and augment their cytotoxic function.. ${ }^{14}$ TRANCE/ TRANCE receptor interaction between $\mathrm{T}$ cell and DC can augment survival of DCs by up regulation of Bcl-XL expression in DC. ${ }^{15} \mathrm{DCs}$ can also cross-present antigens, i.e. present exogenous antigens on their MHC class I molecules to $\mathrm{CD} 8^{+} \mathrm{T}_{\text {cells. }}{ }^{16}$

DCs can induce various types of helper $\mathrm{T}$ cell responses. Upon ligation of PRRs to pathogenic structures and danger signaling, DCs become activated and produce several pro inflammatory cytokines, such as IL-6, TNF- $\alpha$, and type I interferons. ${ }^{17}$ Pro inflammatory cytokines secreted by DCs can favor Th2 directed responses. IL-6 secretion by DCs directs the differentiation of IL-4 producing $\mathrm{CD}^{+} \mathrm{T}$ cells ${ }^{18}$ and impedes Th1 immune responses. ${ }^{19}$ In contrast, IFN- $\alpha$ inhibits IL-4 driven Th2 differentiation and reverses Th2 commitment and stability by suppressing of GATA3 expression..$^{20}$ DCs also favor induction of $\mathrm{T}$ responses toward Th1-type and give rise to higheraffinity $\mathrm{T}$ cells. ${ }^{21}$ Mature DCs can produce high levels of IL-12, a major cytokine in the polarization of naïve $\mathrm{CD}^{+} \mathrm{T}$ cells toward $\mathrm{Th} 1$ cells. IL-12 activates STAT4 signaling pathway and the transcriptional factor Tbet, which are involved in the differentiation of naïve $\mathrm{CD}^{+} \mathrm{T}$ cells into Th1 cells. This cytokine also activates NK cells, a major cell population with antitumor activities. ${ }^{22}$

\section{Generation of tumor-specific T cells with DC vaccines}

DCs can be used to appropriately activate $\mathrm{T}$ cells and induce antigen-specific antitumor immune responses both in vitro and in vivo. Tumor reactive $\mathrm{T}$ cell clones have been generated by repeated antigen-specific stimulation of T cells with DCs in vitro. In a pilot study in leukemia patients, $\mathrm{CD} 8^{+} \mathrm{T}$ cell clones reactive to Wilms tumor antigen 1 (WT1) were generated by repeated stimulation of allogeneic $\mathrm{CD} 8^{+} \mathrm{T}$ cells with peptide-pulsed autologous DCs in vitro. After adoptive transfer of these $\mathrm{T}$ cell clones to leukemia patients who had been received hematopoietic stem cell transplantation, longterm persistence of transferred cells in the peripheral blood and anti leukemic activity were observed in some patients; two of 11 patients showed transient responses and three patients showed stable disease. ${ }^{23}$ Since 1990s, ex vivo generated DCs pulsed with tumor antigens were used to induce antitumor immune responses in murine tumor models. In preliminary studies, it was reported that vaccination with DCs pulsed with peptides or soluble protein results in generation of tumor antigen-specific cytotoxic T cells. ${ }^{24,25}$ Antitumor effects of peptide-pulsed DCs were demonstrated to be dependent on T cells, signals from co stimulatory molecules B7, and Th1 cytokines. ${ }^{26}$ Tumor specific antigens are not recognized in most tumors/cancers. Thus, total antigen resources have been widely used for antigen loading of ex vivo generated DCs in most types of tumors/cancers. Additionally, it is supposed that total tumor antigen loaded DC vaccines contain both MHC class I- and class II-restricted epitopes, hence, these vaccines can induce multivalent $\mathrm{CD}^{+}$and $\mathrm{CD}^{+} \mathrm{T}$ cell responses. In most animal studies, tumor cell lysates have been used as tumor antigens source for antigen-loading of DCs in various tumor models. Stimulation of T cells by tumor lysate-pulsed DCs led to generation of tumor cell-specific cytotoxic T cells (CTLs) in mice. ${ }^{27}$ In a myeloma mouse model, vaccination with DCs pulsed with idiotype or tumor lysate induced anti-idiotype and anti-tumor lysate antibodies, myeloma specific CTLs, Th1 cells, and memory T cells. However, tumor lysate-pulsed DC vaccine was reported to be more potent than idiotype-pulsed DC vaccine in promotion of antitumor immunity. ${ }^{28}$ In general, tumor cell lysate-pulsed DCs produced better clinical responses than highly specific tumor vaccines. ${ }^{29}$ Recently, DC loading of cancer stem cell lysate was reported to be more effective than DCs loaded with unsorted total tumor lysate in the induction of antitumor immunity. Splenocytes isolated from the host vaccinated with DCs pulsed with unsorted total tumor lysate produced higher amount of IFN- $\gamma$ and GM-CSF than splenocytes isolated from host vaccinated with DCs pulsed with unsorted total tumor lysate in murine melanoma and squamous cell cancer models. ${ }^{30}$ Other approaches, including tumor cell RNA, apoptotic tumor cell, DC fusion to tumor cells, and tumor-derived exosomes, have also been used for antigenloading of DCs. Total tumor mRNA-electro prorated DCs were more potent than total tumor lysate-electroporated DCs in the induction of antitumor immune responses in vitro and suppression of tumor growth in MC-38-carcinoembryonic antigen colon cancer-bearing mice. ${ }^{31}$ DCs loaded with tumor cell-derived exosomes could activate tumorspecific CTLs. ${ }^{32}$ Exosomes secreted from DCs may contain molecules involved in immune responses such as MHC, costimulatory, and adhesion molecules as well as heat shock proteins. Exosomes derived from DCs pulsed with peptides induced antitumor immune responses in mice. ${ }^{33}$ Combinational immunotherapy is beneficial to augment DC vaccine-induced antitumor $\mathrm{T}$ cell immune responses, especially in poorly immunologic tumors. For example, administration of antiCD137 (4-1BB) monoclonal antibody after tumor lysate pulsed DCs augments the antitumor efficacy of DC vaccine in murine tumor models. ${ }^{34}$

\section{Clinical effects of DCs vaccines}

In the past decade, monocyte-derived DC vaccines have been used in cancer patients in several clinical trials. In a phase I trial in 14 patients with stage IV solid tumors, including melanoma, neuro blastoma, and colorectal cancer, intradermal vaccination with tumor lysate pulsed DCs induced immunological responses 
in most vaccinated patients without grade 3 or 4 toxicity or visible autoimmunity. Local accumulation of $\mathrm{CD} 4^{+}$and $\mathrm{CD} 8^{+} \mathrm{T}$ cells was observed the injection site. However, therapeutic response was not observed in most vaccinated patients. ${ }^{35}$ In a pilot study in 15 patients with metastatic cancers, including 10 melanoma, two lung cancer, one renal cell carcinoma, one sarcoma, and one breast cancer, autologous tumor lysate-pulsed DCs followed by subcutaneous injection of IL-2, IFN- $\alpha$, and GM-CSF daily for 10days. Therapy was repeated every 21 days for three courses. But, clinical responses were minor. ${ }^{36}$ In a phase I/II trial in patients with renal cell carcinoma (six patients) or breast cancer (four patients), vaccination with autologous tumor lysate-pulsed DCs differentiated from $\mathrm{CD} 34^{+}$hematopoietic stem cells in the presence of GM-CSF/IFN- $\gamma$ followed by administration of low-dose IL-2 produced antigen specific immune responses in most vaccinated patients. DC vaccine in combination with administration of IL-2 was well tolerated. However, clinical response was only observed in one renal cell carcinoma patient as stable disease. ${ }^{37}$ DC vaccine efficacy was also not satisfactory in most cancer patients in other clinical trial. ${ }^{3}$ In recent clinical trials, various strategies have been investigated to improve therapeutic efficacy of DC vaccines.

\section{Improving of therapeutic efficacy of DC vaccinations in recent clinical trials}

Tumor lysate-pulsed DC vaccines have been used in various malignancies, such as melanoma, renal cell carcinoma, cutaneous $T$ cell lymphoma, prostate cancer, ovarian cancer, and leukemia. Sipuleucel-T is the only DC vaccine which was approved by the U.S. FDA in 2010 to treat patients with asymptomatic, or minimally symptomatic, castrateresistant (hormone refractory) metastatic prostate cancer. This vaccine is composed of autologous ex vivo generated DCs pulsed with the antigen "prostatic acid phosphatase (PAP)". ${ }^{38}$ In a phase I clinical trial, injection of monocytes-derived DCs loaded with p53 peptide, which is accumulated in head and neck squamous cell carcinoma (HNSCC) cells, into inguinal lymph nodes of patients with HNSCC resulted in high frequencies of p53-specific T cells in $69 \%$ of patients and IFN- $\gamma$ secretion in $25 \%$ of patients. In addition, vaccination led to decreased Treg levels. Two-year disease free survival was observed in $88 \%$ of vaccinated patients. ${ }^{39}$ In another study, intranodal vaccination with apoptotic tumor cell-loaded DCs was well tolerated in all vaccinated patients with stage III/IV HNSCC, and disease-free survival was more than five years. ${ }^{40}$ Vaccination with hypochlorous acid-oxidized tumor lysate-pulsed DCs reduced circulating Tregs and elicited potent $\mathrm{T}$ cell responses against ovarian antigens in patients with recurrent ovarian cancer. Two of five vaccinated patients experienced durable progression-free survival of two years and more. ${ }^{41}$ Targeting $\mathrm{CD}^{+}$Th cells with DCs pulsed with both MHC class I and MHC class II- restricted epitopes improved induction of antitumor immune responses of the DC vaccine in melanoma patients. ${ }^{42} \mathrm{PD}-\mathrm{L}$-silenced and antigen mRNA-loaded DCs superiorly boosted ex vivo antigenspecific $\mathrm{CD}^{+} \mathrm{T}$ cell responses from transplanted cancer patients without affecting migratory capacity of DCs. ${ }^{43}$ Injection a tetanus/ diphtheria toxoid in the vaccine site one day before vaccination with DCs pulsed with Cytomegalovirus phosphoprotein 65 RNA resulted in improved lymph node migration of DCs and prolonged survival of patients with glioblastoma. Similarly, preconditioning the vaccine site with a shot of tetanus/diphtheria toxoid before DC vaccination significantly promoted the migration of DCs to the lymph node and improved the efficacy of DC vaccine in mice. ${ }^{44}$ Targeting of antigen to DEC-205, the DC receptor for endosytosis, can increase antigen presentation via MHC class II-positive lysosomal compartments. ${ }^{45}$ However, targeting of protein antigen to the DEC-205 can induce
T cell tolerance. ${ }^{46}$ Addition of adjuvants such as TLR ligands to DEC-205-targeting vaccines can avoid antigen-specific tolerance. ${ }^{47}$ In a phase 1 trial in patients with advanced malignancies refractory to available therapies, delivery of the tumor associated antigen NYESO-1 to DCs using the monoclonal antibody CDX-1401, specific for DEC-205 and fused to the antigen NY-ESO-1, in combination with TLR agonists resiquimod (TLR7/8) and Hiltonol (Poly-ICLC, TLR3) elicited robust antigen-specific immune responses in patients with NY-ESO-1-expressing tumors. Thirteen of 45 treated patients showed stabilization of disease, and two patients showed objective tumor regression. ${ }^{48}$

Vaccination with tumor lysate-pulsed DCs in combination with adoptive transfer of ex vivo-activated T cells after curative surgery improved recurrence-free survival and overall survival of patients with invasive hepato cellular carcinoma ${ }^{49}$ Adoptive transfer of autologous MART-1 peptide-pulsed DCs together with TCR transgenic T cells resulted in tumor regression in nine of 13 treated patients. ${ }^{50}$ It has also been found that vaccination with DCs containing lymphocytes during DC culture elicited increased immunogenicity (proliferation of $\mathrm{T}$ cells) in patients with prostate cancer or primary brain tumors. These lymphocytes were proliferating and producing IFN- $\gamma$ in response to antigen in vitro at the time of administration. For preparation of this DC vaccine, immature DCs generated ex vivo by adherence method, in which up to $10 \%$ lymphocytes were also present together with DCs, were co cultured with UV irradiated tumor cells and matured in the presence of PGE2 and TNF- $\alpha .{ }^{51}$

\section{Obstacles in the generation of effective antitumor immunity upon DC vaccination}

Poor immunogenicity of tumor cells is an important obstacle in the induction of antitumor immune responses. Antitumor immune responses of DCs and $\mathrm{T}$ cells can also be suppressed by tumor cells, tumor stromal cells, and various immunosuppressive cells such as Tregs, myeloid derived suppressor cells, tolerogenic DCs, macrophages, NK cells, and NKT cells. In addition, inadequate numbers of lymphocytes in the tumor site due to low persistence, and low efficiency to migrate and infiltrate to tumor tissue are involved in poor antitumor effectiveness of lymphocytes. Overcoming these obstacles can improve efficacy of anticancer DC vaccines. Combinational immunotherapy can augment antitumor efficacy of DC vaccines. However, some therapies used in combination therapy may lead to toxicity or opposite effects. For example, administration of IL-2 together with DC vaccination led to increased frequencies of Tregs in the peripheral blood. ${ }^{52}$ Grade 3 adverse immunologic reaction was reported in a patient with glioblastoma after administration of autologous RNA-pulsed DC vaccines combined with GM-CSF and dose-intensified temozolomide due to the sensitization to the GM-CSF and production of high levels of anti-GM-CSF auto antibodies during vaccination..$^{53}$ Conditioning vaccination site with irradiated MIP- $3 \alpha-$ transfected tumor cells prior to injection of DCs enhanced antitumor efficacy of DC vaccine in a melanoma tumor model..$^{54}$ However, coexpression of MIP- $1 \alpha$ antagonized the GM-CSF-induced antitumor immune responses of subcutaneous GM-CSF-stimulated DCs in a mouse glioma model..$^{55}$ Thus, it should be considered to avoid toxicity or opposite effects in combinational therapies. Repeated vaccination with antigen-loaded DCs may result in elimination of injected DCs by antigen-specific CTLs in vivo. ${ }^{56}$ Therefore, Appropriate DC injection intervals should be considered. The frequency of DC vaccine injections should also be taken into account, because overstimulation can result in terminal differentiation and activation induced cell death (AICD) or exhaustion of T cells. ${ }^{57}$ 
Immunotolerogenic/immunosuppressive DCs may be generated during DC vaccine preparation. Tolerogenic DCs express the immunosuppressive molecules such as PD-L1, PD-L2, CD95L, TRAIL, galectin-1, and CTLA-4, and secrete IL-10, TGF- $\beta$, IDO, IL-27, arginase-1, heme oxigenase-1, and NO. Various agents, including different microbial products, cytokines, growth factors, and inflammatory mediators have been used for induction of maturation in ex vivo generated DCs. But, some of these factors can induce tolerogenic DCs. For example, a cocktail of PGE2 and inflammatory cytokines IL-1 $\beta$, IL- 6 , and TNF- $\alpha$ has been used for maturation of DCs in several studies. But, these maturation stimuli lead to decreased production of IL-12 by DCs and low anticancer effectiveness of DC vaccine. ${ }^{3}$ Some of microbial products also induce immunosuppressive DCs. In a previous work, we observed that DCs matured in the presence of inactivated Lactobacillus crispatus produced high levels of IL-10 whereas IL-12 production was at low levels in an inverse dose-dependent manner, and induced $\mathrm{T}$ cells polarization toward $\mathrm{CD}^{+} \mathrm{CD} 25^{+} \mathrm{FOXP} 3^{+}$Tregs. $^{58}$ Tolerogenic DCs can also induce type 1 regulatory T cells ( $\operatorname{Tr} 1$ cells) and regulatory B cells. ${ }^{59}$ Additionally, tolerogenic DCs suppress $\mathrm{T}$ cell clonal expansion. ${ }^{60}$ On the other hand, Tregs can induce performing-dependent DC death within tumor-draining lymph nodes. ${ }^{61}$ Antitumor activity of injected DCs may be suppressed at the tumor microenvironment. Tumor microenvironment-derived factors such as VEGF, PGE2, and IL-10 can vigorously affect DCs function. ${ }^{62}$ In our previous study, intratumoral injection of immature DCs resulted in enhanced tumor growth in a breast cancer model. ${ }^{63}$ Appropriate maturation induction in ex vivo generated DCs may improve the therapeutic efficacy of DC vaccines. TLR3 agonists have been used for generation of Th1polarizing DCs secreting IL-12. ${ }^{64}$ Stimulation of DCs with TLR4 and TLR7/8 agonists also led to secretion of IL-12. ${ }^{65}$ Other maturation stimuli can also be used to generate mature DCs with appropriate antitumor activity. Poor migration of injected DCs to peripheral lymphoid tissues may be responsible for low antitumor efficiency of DC vaccines observed in most studies as proper interactions of antigen-bearing DCs and T cells occur within the lymphoid tissues. The majority of injected DCs remains at the site of injection and do not migrate to the draining lymph node. ${ }^{66}$ Migration of tumor antigenpulsed DCs to lymph nodes is related to maturation state of DCs. ${ }^{67}$ Expression of chemokine receptors such as CCR7 in mature DCs is important in the migration of DC into lymph nodes. ${ }^{68}$ In addition, the route of DC vaccination and number of DCs reach the lymph nodes can affect therapeutic efficacy of DC vaccines. It has been shown that elicited $\mathrm{CD}^{+} \mathrm{T}$ cell response in the lymph node is proportional to the number of antigen-bearing DCs reaching the lymph node. ${ }^{69}$ In mice, more DCs were detected in lymph nodes after subcutaneous or intradermal injection compared to intravenous injection. ${ }^{70}$

In another study, a few DCs migrated to draining lymph nodes after subcutaneous injection. ${ }^{71}$ In humans, subcutaneously injected DCs were not found in lymph nodes and less than $1 \%$ of intradermal injected DCs were detected in draining lymph nodes. Only a few DCs injected intradermally were detected in draining lymph nodes. ${ }^{72}$ In patients with melanoma, intravenous injection of peptide-pulsed DCs was reported to reduce the immune responses in the peripheral blood. ${ }^{73}$ In patients with prostate cancer, intradermal, intralymphatic, and intravenous injection of antigen-loaded DCs led to induction of antigen-specific $\mathrm{T}$ cell responses in all patients. However, Th1-related response was only detected after intradermal and intralymphatic injection. ${ }^{74}$ Increasing the number of injected $\mathrm{CCR} 7^{+}$DCs may improve migration of injected DCs into lymph nodes. CCR $7^{+}$DCs efficiently induce increased cellularity in lymph nodes. ${ }^{69}$ In patients with HNSCC, intranodal vaccination with apoptotic tumor cell-loaded DCs was effective in vaccinated patients as disease-free survival was more than five years. ${ }^{40}$ Overcoming these obstacles improves antitumor efficacy of DC vaccines in patients with cancer. Culture conditions and maturation induction in DCs as well as amounts of DCs, number of injections, time of injections, and route of injections can be optimized for efficient DC vaccination.

\section{Conclusion}

DC-based vaccination is safe and can induce antitumor immune responses in patients with cancer. However, clinical responses are partial in most vaccinated cancer patients and more work is needed to improve DC vaccine potency. Identification of tumor antigen specific antigens can improve tumor antigen loading of DCs and facilitate production of tumor specific $\mathrm{T}$ cells. Culture condition, source of tumor antigen, antigen loading strategy, and maturation stimuli are crucial in the persistence and functional constancy of injected DCs and favoring $\mathrm{T}$ cell responses toward Th1-type and expanding of tumorspecific effectors T cells. Optimizing these steps as well as route, dose, and schedule of DC vaccination augments antitumor efficacy of DC vaccines in cancer patients.

\section{Acknowledgments}

None.

\section{Conflicts of interest}

Author declares there are no conflicts of interest.

\section{Funding}

None.

\section{References}

1. Steinman RM, Hemmi H. Dendritic cells: translating innate to adaptive immunity. Curr Top Microbiol Immunol . 2006;311:17-58

2. Bonaccorsi I, Campana S, Morandi B, et al. Acquisition and presentation of tumor antigens by dendritic cells. Crit Rev Immunol . 2015;35(5):349-364.

3. Farashi-Bonab S, Khansari N. Dendritic cell vaccine and its application in cancer therapy. Int J Vaccines Vaccin. 2015;1(1):00002.

4. Banchereau J, Briere F, Caux C, et al. Immunobiology of dendritic cells Annu Rev Immunol. 2000;18:767-811.

5. Vyas JM, Van der Veen AG, Ploegh HL. The known unknowns of antigen processing and presentation. Nat Rev Immunol . 2008;8(8):607-618.

6. Merad M, Manz MG. Dendritic cell homeostasis. Blood. 2009;113(15):3418-3427.

7. Kilinc $\mathrm{MO}, \mathrm{Gu} \mathrm{T}$, Harden JL, et al. Central role of tumor associated $\mathrm{CD}^{+} \mathrm{T}$ effector/memory cells in restoring systemic antitumor immunity. J Immunol . 2009;182(7):4217-4225.

8. Hadrup S, Donia M, Thor Straten P (2013) Effector CD4 and CD8 T cells and their role in the tumor microenvironment. Cancer Microenviron 6 (2): 123-133.

9. Kim HJ, Cantor H. CD4 T-cell subsets and tumor immunity: the helpful and the not-so-helpful. Cancer Immunol Res. 2014;2(2):91-98.

10. Sallusto F, Lanzavecchia A. The instructive role of dendritic cells on T-cell responses. Arthritis Res. 2002;4 (Suppl 3):S127-S132.

11. Nakayama T, Yamashita M. The TCR-mediated signaling pathways that control the direction of helper $\mathrm{T}$ cell differentiation. Semin Immunol .2010;22(5):303-309. 
12. Ballesteros-Tato

A,

Randall

TD

Priming of T follicular helper cells by dendritic cells. Immunol Cell Biol. 2014;92(1):22-27.

13. Coquet JM, Rausch L, Borst J. Priming of T follicular helper cells by dendritic cells. Immunol Cell Biol. 2015;93(9):780-788.

14. Schoenberger SP, Toes RE, van der Voort EI, et al. T-cell help for cytotoxic $\mathrm{T}$ lymphocytes is mediated by $\mathrm{CD} 40-\mathrm{CD} 40 \mathrm{~L}$ interactions. Nature. 1998;393(6684):480-483.

15. Wong BR, Josien R, Lee SY, et al. TRANCE (tumor necrosis factor [TNF]-related activation-induced cytokine), a new TNF family member predominantly expressed in T cells, is a dendritic cell-specific survival factor. $J$ Exp Med. 1997;186(12):2075-2080.

16. Joffre OP, Segura E, Savina A, et al. Cross-presentation by dendritic cells. Nat Rev Immunol. 2012;12(8):557-569.

17. Gorelik M, Frischmeyer-Guerrerio PA. Innate and adaptive dendritic cell responses to immunotherapy. Curr Opin Allergy Clin Immunol. 2015; 15(6):575-580

18. Rincón M, Anguita J, Nakamura T, et al. Interleukin (IL)-6 directs the differentiation of IL-4-producing $\mathrm{CD}^{+} \mathrm{T}$ cells. $J$ Exper Med. 1997;185(3):461-469.

19. Dodge IL, Carr MW, Cernadas M, et al. IL-6 production by pulmonary dendritic cells impedes Th1 immune responses. $J$ Immunol. 2003;170(9):4457-4464.

20. Huber JP, Ramos HJ, Gill MA, et al. Cutting edge: Type I IFN reverses human Th2 commitment and stability by suppressing GATA3. J Immunol. 2010;185(2):813-817.

21. Tanaka H, Demeure CE, Rubio M, et al. Human monocyte-derived dendritic cells induce naive $\mathrm{T}$ cell differentiation into $\mathrm{T}$ helper cell type 2 (Th2) or Th1/Th2 effectors. J Exp Med. 2000; 192(3):405-412.

22. Thieu VT, Yu Q, Chang HC, et al. Stat4 is required for T-bet to promote IL-12-dependent Th1 fate determination. Immunity. 2008;29(5):679-690.

23. Chapuis AG, Ragnarsson GB, Nguyen HN, et al. Transferred WT1 reactive $\mathrm{CD} 8^{+} \mathrm{T}$ cells can mediate antileukemic activity and persist in post-transplant patients. Sci Transl Med. 2013;5:(174) 174ra27.

24. Celluzzi CM, Mayordomo JI, Storkus WJ, et al. Peptide-pulsed dendritic cells induce antigen-specific CTL-mediated protective tumor immunity. $J$ Exp Med. 1996;183(1):283-287.

25. Paglia $\mathrm{P}$, Chiodoni C, Rodolfo M, et al. Murine dendritic cells loaded in vitro with soluble protein prime cytotoxic $\mathrm{T}$ lymphocytes against tumor antigen in vivo. $J$ Exp Med. 1996;183(1):317-322.

26. Zitvogel L, Mayordomo JI, Tjandrawan T, et al. Therapy of murine tumors with tumor peptide-pulsed dendritic cells: dependence on $\mathrm{T}$ cells, B7 costimulation, and T helper cell 1-associated cytokines. J Exp Med. 1996;183(1):87-97.

27. Ghosh A, Wolenski M, Klein C, et al. Cytotoxic T cells reactive to an immunodominant leukemia-associated antigen can be specifically primed and expanded by combining a specific priming step with nonspecific large-scale expansion. J Immunother. 2008;31(2):121-131.

28. Hong S, Li H, Qian J, et al. Optimizing dendritic cell vaccine for immunotherapy in multiple myeloma: tumour lysates are more potent tumour antigens than idiotype protein to promote anti-tumour immunity. Clin Exp Immunol . 2012;170(2):167-177.

29. Neller MA, López JA, Schmidt CW. Antigens for cancer immunotherapy. Seminv Immunol. 2008;20(5):286-295

30. Li Q, Lu L, Tao H, et al. Generation of a novel dendritic-cell vaccine using melanoma and squamous cancer stem cells. J Vis Exp. 2014;83:e50561.

31. Osada T, Nagaoka K, Takahara M, et al. Precision cancer immunotherapy: optimizing dendritic cell-based strategies to induce tumor antigenspecific t-cell responses against individual patient tumors. J Immunother. 2015; 38(4): 155-164

32. Wolfers J, Lozier A, Raposo G, et al. Tumor-derived exosomes are a source of shared tumor rejection antigens for CTL cross-priming. Nat Med. 2001;7(3):297-303.

33. Zitvogel L, Regnault A, Lozier A, et al. Eradication of established murine tumors using a novel cell-free vaccine: dendritic cell-derived exosomes. Nat Med. 1998;4(5):594-600

34. Ito $\mathrm{F}, \mathrm{Li} \mathrm{Q}$, Shreiner $\mathrm{AB}$, et al. Anti-CD137 monoclonal antibody administration augments the antitumor efficacy of dendritic cell-based vaccines. Cancer Res. 2004;64(22): 8411-8419.

35. Chang AE, Redman BG, Whitfield JR, et al. A phase I trial of tumor lysate-pulsed dendritic cells in the treatment of advanced cancer. Clin Cancer Res. 2002;8(4):1021-1032.

36. Mayordomo JI, Andres R, Isla MD, et al. Results of a pilot trial of immunotherapy with dendritic cells pulsed with autologous tumor lysates in patients with advanced cancer. Tumori . 2007;93(1): 26-30.

37. Baek S, Kim CS, Kim SB, et al. Combination therapy of renal cel carcinoma or breast cancer patients with dendritic cell vaccine and IL-2: results from a phase I/II trial. $J$ Transl Med. 2011; 9: 178.

38. Kantoff PW, Higano CS, Shore ND, et al. Sipuleucel-T immunotherapy for castration-resistant prostate cancer. $N$ Engl J Med . 2010;363(5):411-422.

39. Schuler PJ, Harasymczuk M, Visus C, et al. Phase I dendritic cell p53 peptide vaccine for head and neck cancer. Clin Cancer Res. 2014;20(9):2433-2444

40. Whiteside TL, Ferris RL, Szczepanski M, et al. Dendritic cell-based autologous tumor vaccines for head and neck squamous cell carcinoma: Promise vs reality. Head Neck Suppl1: E4. 2016;94-501.

41. Chiang CL, Kandalaft LE, Tanyi J, et al. A dendritic cell vaccine pulsed with autologous hypochlorous acid-oxidized ovarian cancer lysate primes effective broad antitumor immunity: from bench to bedside. Clin Cancer Res. 2013;19(17):4801-4815.

42. Aarntzen EH, De Vries IJ, Lesterhuis WJ, Schuurhuis D, Jacobs JF, et al. (2013) Targeting CD4 $\left(^{+}\right)$T-helper cells improves the induction of antitumor responses in dendritic cell-based vaccination. Cancer Res 73(1): 19-29.

43. Hobo W, Novobrantseva TI, Fredrix H, Wong J, Milstein S, et al. (2013) Improving dendritic cell vaccine immunogenicity by silencing PD-1 ligands using siRNA-lipid nanoparticles combined with antigen mRNA electroporation. Cancer Immunol Immunother 62(2): 285-297.

44. Mitchell DA, Batich KA, Gunn MD, Huang MN4, Sanchez-Perez L, et al. (2015) Tetanus toxoid and CCL3 improve dendritic cell vaccines in mice and glioblastoma patients. Nature 519(7543): 366-369.

45. Mahnke K, Guo M, Lee S, et al. The dendritic cell receptor for endocytosis, DEC-205, can recycle and enhance antigen presentation via major histocompatibility complex class II-positive lysosomal compartments. $J$ Cell Biol. 2000;151:(3):673-684.

46. Bonifaz L, Bonnyay D, Mahnke K, et al.. Efficient targeting of protein antigen to the dendritic cell receptor DEC-205 in the steady state leads to antigen presentation on major histocompatibility complex class I products and peripheral $\mathrm{CD} 8^{+} \mathrm{T}$ cell tolerance. $J$ Exp Med. 2002;196(12):1627-1638

47. Kreutz M, Giquel B, Hu Q, et al. Antibody-antigen-adjuvant conjugates enable co-delivery of antigen and adjuvant to dendritic cells in cis but only have partial targeting specificity. PLoS One. 2010;7(7):e40208.

48. Dhodapkar MV, Sznol M, Zhao B, et al. Induction of antigen-specific immunity with a vaccine targeting NY-ESO-1 to the dendritic cell receptor DEC-205. Sci Transl Med. 2014;6(232):232ra51. 
49. Shimizu K, Kotera Y, Aruga A, et al. Postoperative dendritic cell vaccine plus activated T-cell transfer improves the survival of patients with invasive hepatocellular carcinoma. Hum Vaccin Immunother. 2014;10(4):970-976.

50. Chodon T, Comin-Anduix B, Chmielowski B, et al. Adoptive transfer of MART-1 T-cell receptor transgenic lymphocytes and dendritic cell vaccination in patients with metastatic melanoma. Clin Cancer Res . 2014;20(9):2457-2465

51. Frank MO, Kaufman J, Parveen S, et al. Dendritic cell vaccines containing lymphocytes produce improved immunogenicity in patients with cancer. $J$ Transl Med. 2014;12:338.

52. Lesterhuis WJ, de Vries IJ, Schreibelt G, et al. Route of administration modulates the induction of dendritic cell vaccine-induced antigenspecific $\mathrm{T}$ cells in advanced melanoma patients. Clin Cancer Res. 2011;17(17):5725-5735.

53. Mitchell DA, Sayour EJ, Reap E, et al. Severe adverse immunologic reaction in a patient with glioblastoma receiving autologous dendritic cell vaccines combined with GMCSF and dose-intensified temozolomide. Cancer Immunol Res. 2015;3(4):320-325.

54. Shih NY, Yang HY, Cheng HT, et al. Conditioning vaccination site with irradiated MIP-3alpha-transfected tumor cells enhances efficacy of dendritic cell-based cancer vaccine. J Immunother. 2009;32(4):363-369.

55. Herrlinger U, Aulwurm S, Strik H, et al. MIP-1alpha antagonizes the effect of a GM-CSF-enhanced subcutaneous vaccine in a mouse glioma model. J Neurooncol . 2004;66(1-2):147-154.

56. Yang J, Huck SP, McHugh RS, et al. Perforin-dependent elimination of dendritic cells regulates the expansion of antigen-specific $\mathrm{CD} 8^{+} \mathrm{T}$ cells in vivo. Proc Natl Acad Sci U S A. 2006; 103(1):147-152.

57. Wherry E. T cell exhaustion. Nat Immunol . 2011;12(6):492-499.

58. Eslami S, Hadjati J, Motevaseli E, et al. Lactobacillus crispatus strain SJ-3C-US induces human dendritic cells (DCs) maturation and confers an anti-inflammatory phenotype to DCs. APMIS . 2016;124(8): 697-710.

59. Volchenkov R, Karlsen M, Jonsson R, et al. Type 1 regulatory T cells and regulatory B cells induced by tolerogenic dendritic cells. Scand J Immunol . 2013;77(4):246-254

60. Mellor AL, Baban B, Chandler P, et al. Cutting edge: induced indoleamine 2, 3 dioxygenase expression in dendritic cell subsets suppresses $\mathrm{T}$ cell clonal expansion. J Immunol. 2003;171(4):1652-1655.

61. Boissonnas A, Scholer-Dahirel A, Simon-Blancal V, et al. Foxp $3^{+}$T cells induce perforin-dependent dendritic cell death in tumor-draining lymph nodes. Immunity. 2010;32(2):266-278.
62. Liu Q, Zhang C, Sun A, et al. Tumor-educated CD11bhighIalow regulatory dendritic cells suppress $\mathrm{T}$ cell response through arginase I. J Immunol . 2009;182(10):6207-6216.

63. Farashi-Bonab S, Khansari N. Dendritic cell maturation is a critical step in dendritic cell vaccine preparation for cancer therapy. Vaccin Res Open J. 2016;1(1):25-32.

64. Mailliard RB, Wankowicz-Kalinska A, Cai Q, et al. $\alpha$-Type-1 polarized dendritic cells: a novel immunization tool with optimized CTL inducing activity. Cancer Res . 2004;64(17):5934-5937.

65. Dauer M, Lam V, Arnold H, et al. Combined use of toll-like receptor agonists and prostaglandin E(2) in the FastDC model: rapid generation of human monocyte-derived dendritic cells capable of migration and IL12p70 production. J Immunol Methods . 2008;337(2):97-105.

66. Ridolfi R, Riccobon A, Galassi R, et al. Evaluation of in vivo labelled dendritic cell migration in cancer patients. J Transl Med. 2004;2(1):1-27.

67. De Vries IJ, Krooshoop DJ, Scharenborg NM, et al. Effective migration of antigen-pulsed dendritic cells to lymph nodes in melanoma patients is determined by their maturation state. Cancer Res. 2003;63(1):12-17.

68. Chen YZ, Ruan GX, Yao XL, et al. Co-transfection gene delivery of dendritic cells induced effective lymph node targeting and antitumor vaccination. Pharm Res. 2003;30(6):1502-1512.

69. MartIn-Fontecha A, Sebastiani S, Höpken UE, et al. Regulation of dendritic cell migration to the draining lymph node: impact on $\mathrm{T}$ lymphocyte traffic and priming. J Exp Med. 2003;198(4):615-621.

70. Eggert AA, Schreurs MW, Boerman OC, et al. Biodistribution and vaccine efficiency of murine dendritic cells are dependent on the route of administration. Cancer Res. 1999;59(14):3340-3345.

71. Josien R, Li HL, Ingulli E,et al. TRANCE, a tumor necrosis factor family member, enhances the longevity and adjuvant properties of dendritic cells in vivo. J Exp Med. 2000;191(3):495-502.

72. Morse MA, Coleman RE, Akabani G, et al. Migration of human dendritic cells after injection in patients with metastatic malignancies. Cancer Res. 1999;59(1):56-58.

73. Thurner B, Röder C, Dieckmann D, et al. Generation of large numbers of fully mature and stable dendritic cells from leukapheresis products for clinical application. J Immunol Methods. 1999;223(1):1-15.

74. Fong L, Brockstedt D, Benike C, et al. Dendritic cells injected via different routes induce immunity in cancer patients. $J$ Immunol. 2001;166(6):4254-4259. 